\title{
Effects of parallel planning on agreement production
}

\author{
Alma Veenstra ${ }^{\mathrm{a}, *}$, Antje S. Meyer ${ }^{\mathrm{b}}$, Daniel J. Acheson ${ }^{\mathrm{b}}$ \\ a Université Libre de Bruxelles, Brussels, Belgium \\ b Max Planck Institute for Psycholinguistics, Radboud University, Nijmegen, the Netherlands
}

\section{A R T I C L E I N F O}

\section{Article history:}

Received 9 February 2015

Received in revised form 31 August 2015

Accepted 26 September 2015

Available online $\mathrm{xxxx}$

\section{Keywords:}

Language production

Subject-verb agreement

Advance planning

Grammatical encoding

Eye movements

\begin{abstract}
A B S T R A C T
An important issue in current psycholinguistics is how the time course of utterance planning affects the generation of grammatical structures. The current study investigated the influence of parallel activation of the components of complex noun phrases on the generation of subject-verb agreement. Specifically, the lexical interference account (Gillespie \& Pearlmutter, 2011b; Solomon \& Pearlmutter, 2004) predicts more agreement errors (i.e., attraction) for subject phrases in which the head and local noun mismatch in number (e.g., the apple next to the pears) when nouns are planned in parallel than when they are planned in sequence.

We used a speeded picture description task that yielded sentences such as the apple next to the pears is red. The objects mentioned in the noun phrase were either semantically related or unrelated. To induce agreement errors, pictures sometimes mismatched in number. In order to manipulate the likelihood of parallel processing of the objects and to test the hypothesized relationship between parallel processing and the rate of agreement errors, the pictures were either placed close together or far apart.

Analyses of the participants' eye movements and speech onset latencies indicated slower processing of the first object and stronger interference from the related (compared to the unrelated) second object in the close than in the far condition. Analyses of the agreement errors yielded an attraction effect, with more errors in mismatching than in matching conditions. However, the magnitude of the attraction effect did not differ across the close and far conditions. Thus, spatial proximity encouraged parallel processing of the pictures, which led to interference of the associated conceptual and/or lexical representation, but, contrary to the prediction, it did not lead to more attraction errors.
\end{abstract}

(c) 2015 Elsevier B.V. All rights reserved.

\section{Introduction}

Language production requires us to translate concepts into words and to use grammatical structures to order these words. During these processes of utterance planning it is often the case that several concepts and the associated words are simultaneously activated in the speaker's mind and compete for inclusion in the utterance plan (Konopka, 2012; Levelt, Roelofs, \& Meyer, 1999; Martin, Crowther, Knight, Tamborello, \& Yang, 2010). A significant amount of research has shown that when words with similar meanings are planned in parallel, semantic interference may occur (Aristei, Zwitserlood, \& Abdel Rahman, 2012; Belke, Meyer, \& Damian, 2005; Glaser \& Glaser, 1989). In contrast, parallel planning of similar speech sounds during phonological encoding can lead to facilitation (Dell, 1986; Humphreys, Boyd, \& Watter, 2010; Oppermann, Jescheniak, \& Schriefers, 2010). Although competing grammatical number forms (as in "the apple next to the pears" vs. "the apple next to the pear") do lead to increased error rates in subject-verb

\footnotetext{
* Corresponding author at: Université Libre de Bruxelles, Avenue F. D. Roosevelt 50, 1050 Brussels, Belgium.

E-mail address: aveenstr@ulb.ac.be (A. Veenstra).
}

agreement (i.e., the 'attraction effect'; Bock \& Miller, 1991), little is known about whether parallel processing per se is the culprit. Part of the reason for the lack of progress on this issue may be methodological as suitable paradigms and measurements have yet to be developed to assess the amount of parallel planning during grammatical encoding. The current study addresses this limitation through use of eyetracking in a picture description paradigm in which we directly assess whether parallel activation of competing number forms influences subject-verb number agreement.

Subject-verb agreement is a central aspect of grammatical encoding in many languages as almost all utterances include verbs that have to be inflected according to the agreement rules of the language. The production of subject-verb agreement can be influenced by syntactic and semantic factors. A robust syntactic influence is the attraction from a local noun that is located between the head noun of a subject phrase and the inflected verb. Attraction-typically studied with sentence completion tasks-occurs when a plural local noun follows a singular subject head noun, increasing the chance for the verb to obtain an incorrect plural inflection (e.g., the key to the cabinets are missing; Bock \& Miller, 1991, see also Bock \& Eberhard, 1993; Bock, Eberhard, Cutting, Meyer, \& Schriefers, 2001; Franck, Vigliocco, \& Nicol, 2002; Haskell, Thornton, \& MacDonald, 2010; Vigliocco, Butterworth, \& Semenza, 1995). To a 
lesser extent the same occurs for plural head nouns combined with singular local nouns (Eberhard, 1997; Veenstra, Acheson, \& Meyer, 2014).

Solomon and Pearlmutter (2004) linked the attraction effect to the time course of sentence planning. When the nouns of a subject phrase are planned in parallel rather than sequentially, their number features are active in working memory simultaneously, giving rise to number interference and agreement errors. This view is supported by a number of experiments varying the degree of semantic integration of the subject noun phrase. Semantic integration is the degree to which a head noun and local noun are related to each other at the conceptual level. The bowl with the spoons is an example of a weakly integrated subject: the bowl and the spoons coexist, but are independent of each other. The bowl with the stripes is an example of a tightly integrated subject: the stripes are part of the bowl. In five sentence completion experiments, Solomon and Pearlmutter found more attraction for integrated than unintegrated subject phrases. They proposed that tight semantic integration encouraged parallel planning and led to more agreement errors relative to weak semantic integration.

Gillespie and Pearlmutter (2011b) also argued for a lexical interference account of attraction, although they referred to it as a scope of planning account. (As both accounts are based on the same assumptions, we will refer to them collectively as a lexical interference account). This account proposes that the strength of the attraction effect is determined by the relative timing of advance planning of the head and local noun. When a local noun that mismatches in number from a head noun is planned simultaneously with the head noun, more attraction errors occur compared to when the nouns are planned sequentially. The likelihood of parallel planning may depend on different factors. Parallel planning can be encouraged by semantic integration, but also by a short linear distance between the nouns. Gillespie and Pearlmutter (2011b) conducted two sentence completion experiments, factorially manipulating the hierarchical and linear distance of two local nouns from a head noun with the semantic integration with the head noun (e.g., the book with the torn pages by the red pen). The authors found that more agreement errors were made for mismatching local nouns that were linearly (and not hierarchically) close to the head noun compared to nouns that were farther apart. The distance and integration factors also interacted as there were even more errors when the nouns were both linearly close and semantically integrated. The authors interpreted their findings to suggest that both semantic integration and linear distance to the head noun affect how likely the head and local noun are planned in parallel, which in turn affects the magnitude of the attraction effect.

However, a follow-up experiment using a picture description task failed to replicate these error patterns (Gillespie \& Pearlmutter, 2011a). Here, participants described object pairs in noun phrases using prepositions that depended on the color of the outline around the first picture. If the color was blue, for had to be used (leading to an integrated phrase, e.g., the apple for the pie), if the color was green, near had to be used (leading to an unintegrated phrase, e.g., the apple near the pie). Gillespie and Pearlmutter found no effect of semantic integration on error rates, but speech onset times were shorter for integrated than unintegrated noun phrases. These results were interpreted to suggest that parallel planning occurred in the integrated phrases and not in the unintegrated phrases. The authors argued that planning an entire phrase was faster when all constituents were being planned at the same time compared to when they were planned more sequentially. The authors suggested that the timing of sentence planning might not have differed sufficiently in the integrated and unintegrated phrases to lead to a reliable effect in the error rates.

Contrary to Solomon and Pearlmutter's (2004) results, Brehm and Bock (2013) found more errors for unintegrated (compared to integrated) noun phrases, irrespective of whether the head and local noun matched or mismatched in number. They proposed an alternative hypothesis to account for their error patterns, with a focus on the influence that semantic integration has on the notional number of subject phrases. This notional number account claims that integrated subject phrases tend to be perceived as notionally singular, regardless of whether the local noun is singular or plural, whereas unintegrated subject phrases are perceived as notionally plural. With a singular head noun, integrated subject phrases are predicted to yield fewer agreement errors than unintegrated subject phrases. Consistent with this prediction, Brehm and Bock found fewer errors and shorter response times for integrated sentences such as the bowl with the red stripes compared to unintegrated sentences such as the bowl with the wooden spoons. Supporting evidence for the notional number account comes from studies of agreement in Dutch using different sentence completion tasks (Veenstra \& Acheson, 2015; Veenstra, Acheson, Bock, \& Meyer, 2014). Veenstra and colleagues found more agreement errors for unintegrated than integrated subject phrases. Similar to Brehm and Bock (2013), the integration effect in these studies was independent of the attraction effect.

These results cast some doubt on the lexical interference account of Solomon and Pearlmutter (2004). This account is based on two assumptions: (1) that semantic integration increases parallel planning and (2) that parallel planning increases attraction. There are several possible reasons why inconsistent effects of semantic integration on attraction have been reported: It could be that semantic integration does not increase parallel planning, or that parallel planning does not increase attraction. A third possibility is that neither assumption is correct. In order to gain traction on these issues, and given that the effect of semantic integration is still under debate, the current study focused on the effect of parallel processing on the agreement process.

Despite claims of parallel planning in earlier studies of agreement, independent measures of such planning have never been recorded. The present study was designed to address this limitation by manipulating the amount of parallel activation of head and local nouns. Before relating the error rates to the amount of parallel planning, we first assessed whether interference between the nouns occurred, which would demonstrate that they were accessed in parallel. In the remainder of the introduction we motivate the use of the experimental paradigm and explain the predictions for the dependent measures.

We used a picture description task, which is a fairly natural way to elicit agreement through the production of whole sentences. More traditionally, however, agreement processes have been studied using sentence completion tasks in which speakers only provide a completion (i.e., an inflected verb phrase) to an experimentally-provided noun phrase. One advantage of picture description is that verbal comprehension, an unavoidable component of the sentence completion paradigm, is eliminated. Importantly, previous research using picture description has been able to replicate attraction effects (Gillespie \& Pearlmutter, 2011a), suggesting that object naming tasks are suitable for assessing agreement.

Veenstra, Acheson, and Meyer (2014) also used a picture description task to study agreement. In contrast to Gillespie and Pearlmutter's (2011a) task where pictures only yielded subject noun phrases (e.g., the apple for the pie), in Veenstra et al.'s task participants based their entire utterance on the pictures (e.g., the star next to the circle is blue). In their study, attraction effects were found for sentences with mismatching head and local nouns, both when the head noun was singular, and when it was plural. The current study used an adaptation of Veenstra et al.'s task, both because it allows for tight control over how sentences are completed and because picture description is particularly well-suited to our current goal of assessing interference. By manipulating the spatial configuration of the pictures, we aimed to manipulate the likelihood of parallel processing of the pictures and the amount of interference between the nouns in the subject noun phrase. The degree of interference should manifest itself in the speakers' naming times and eye movements.

Earlier multi-object naming studies have shown that when objects are close together, speakers process them in parallel. For instance, Meyer, Ouellet, and Häcker (2008) conducted a naming task in which 
participants had to name three pictures. When they shifted their eye gaze to the second picture, this picture was replaced with a new picture. Facilitation was found when the old and new picture were identical or homophonous, suggesting that the second (old) picture was processed while the participants were still looking at the first picture (see also Mädebach, Jescheniak, Oppermann, \& Schriefers, 2011; Malpass \& Meyer, 2010; Morgan, Van Elswijk, \& Meyer, 2008; Schotter, Ferreira, \& Rayner, 2013).

In the study by Meyer et al. (2008) the pictures had a size of approximately $5.7^{\circ}$ and were placed at a midpoint-to-midpoint distance of $14.6^{\circ}$. The effect of spatial distance on semantic interference was also addressed by Meyer and Konopka (2011), in a multiple object naming task where pictures either belonged to the same semantic category or were unrelated. Pictures had a size of $2.6^{\circ}$ and were presented in a near $\left(2.7^{\circ}\right.$ midpoint-to-midpoint), middle $\left(9.2^{\circ}\right)$, or far spatial configuration $\left(15.5^{\circ}\right)$. Meyer and Konopka found semantic interference in speech onsets and gaze durations for looks at the first picture in the near and middle conditions, but not in the far condition. The interference effect was stronger in the near than in the middle condition. One account of these semantic interference effects is that the selection of the lemma for the first noun is slowed down by the co-activation of the related second lemma (e.g., Freedman, Martin, \& Biegler, 2004; Smith \& Wheeldon, 2004). Thus, co-activation of a second lemma was more likely in the near and middle conditions, where the second object could be identified while the first one was fixated upon. No such effect was observed in the far condition, where the second picture could not be identified without a shift of gaze towards it.

The study by Meyer and Konopka indicates that the degree of semantic interference between pictures that are to be named in succession can be affected by the spatial configuration of pictures. In the present study we built upon this finding. We presented pictures either spatially close (facilitating parallel activation) or far apart (hindering parallel activation). The pictures were either semantically related or unrelated. We predicted that semantic interference would occur in the close but not in the far condition. This interference would be evidenced in longer speech onsets and longer gaze durations for semantically related compared to unrelated pictures. In contrast to earlier studies, the manipulation of spatial distance and the assessment of semantic interference enabled us to determine whether parallel processing of the two objects occurred. With parallel activation established, we were then able to address whether it influenced the generation of agreement.

To summarize, the key issue addressed in the current study is whether differences in interference between the components of the subject noun phrase were related to differences in subject-verb agreement as assessed through the strength of the attraction effect. Given our manipulations, the lexical interference account (Gillespie \& Pearlmutter, 2011b; Solomon \& Pearlmutter, 2004) predicts stronger attraction in the close than in the far condition.

\section{Method}

\subsection{Participants}

Twenty-four native speakers of Dutch (mean age 23 years, $S D=5.3$ ) participated in the experiment and received $€ 8$ for their participation. Sixteen participants were female; twenty-two were university students. All gave written informed consent prior to the study. Approval to conduct the study was given by the Ethics Board of the Social Sciences Faculty of Radboud University Nijmegen.

\subsection{Materials and design}

Twenty-eight pictures were used in the experiment. To facilitate picture naming, we used objects with highly frequent names. The average natural $\log$ frequency taken from CELEX for the singular forms was 6.3 $(\mathrm{SD}=1.3)$; for the plural forms this was $5.4(\mathrm{SD}=1$; Baayen,
Piepenbrock, \& Van Rijn, 1993). The pictures were taken from the Severens picture database and had a mean naming latency of $901 \mathrm{~ms}$ ( $\mathrm{SD}=145 \mathrm{~ms}$; Severens, Lommel, Ratinckx, \& Hartsuiker, 2005). The pictures were simple black line drawings that were all approximately $128 \times 98$ pixels in size, viewed with $2 \times 1.5^{\circ}$ visual angle. As the small set of items was presented multiple times (each picture appeared 64 times in the experiment), object identification and naming should be relatively easy (e.g., Francis, Corral, Jones, \& Sáenz, 2008; Malpass \& Meyer, 2010).

The experiment utilized a 2 (Head Noun Number: singular/plural) by 2 (Number Mismatch: match/mismatch) by 2 (Semantic Similarity: related/unrelated) by 2 (Spatial Distance: close/far) within-subjects design. Fourteen noun pairs were constructed, consisting of two related nouns belonging to the same semantic category. These pairs were recombined to form an additional fourteen unrelated pairs. For example, the related pairs apple/pear and pants/sweater were recombined into the unrelated pairs apple/sweater and pants/pear. Similarly, boat/ canoe and scarf/hat were recombined into boat/hat and scarf/canoe; see Tables A1 to A3 in the appendix for a list of items and item pairs.

The head noun picture always appeared in the top left corner of the computer screen. The local noun picture was 100 pixels to the right $\left(1.5^{\circ}\right.$ visual angle $)$ of the head noun picture in the close condition, and 800 pixels to the right $\left(12^{\circ}\right.$ visual angle) in the far condition. A colored box was positioned 400 pixels below the local noun picture $\left(6^{\circ}\right.$ visual angle) at the bottom of the screen. See Fig. 1 for an example item.

Meyer, Van Der Meulen, and Brooks (2004) conducted an eyetracking study with similar target sentences (e.g., the chair next to the star is brown). Using a colored first picture, they found that participants often looked back to the first picture when they produced the color word (47\%). In our study it was important that participants did not look back to the first picture after naming it, as they may re-activate the number information of the head noun, thus decreasing the chance of attraction. We accomplished this goal in two ways. First, participants only had 2.5 seconds to complete the entire sentence they were generating, as indicated by a timer in the middle of the screen (see Fig. 1 and below). Second, the color to be used to describe the head noun was indicated not by the head noun itself, but by a separate picture of a small colored box of $23 \times 23$ pixels ( $0.3^{\circ}$ visual angle) positioned at the bottom of the screen underneath the local noun picture (see Fig. 1). The colors used were very pale versions of green, blue, yellow, and red. Because of the large distance from the previous picture, the small size and paleness of the color, the colored box was difficult identify extrafoveally, which encouraged participants to initiate an eye movement and fixate the color picture before naming it.

Each item was combined with four colors, which resulted in a total of 896 items, divided over four experimental lists. Each list was divided into four blocks in which the conditions were equally represented. Each block consisted of 56 trials, in a fixed random order. Twenty additional trials were created as practice trials.

\subsection{Apparatus}

Participants' eye movements were recorded with an SR Research Eye Link 1000 eye tracker with chin rest. The right eye was tracked for all participants at a sampling rate of $1000 \mathrm{~Hz}$. Before each block a 9-point calibration was performed to link the position of the eyes with a location on the screen. Drift correction was performed before each trial to control for head movements between trials. The experiment was programmed with Experiment Builder software, and the eye-tracking data were quantified with Data Viewer, both from SR Research.

The experiment was stored and run on a Dell Precision desktop computer and presented on an Acer AL2023 20-inch LCD monitor (16.6 ms refresh rate, $60 \mathrm{~Hz}$ ). Participants' responses were recorded with a Sennheiser microphone. 


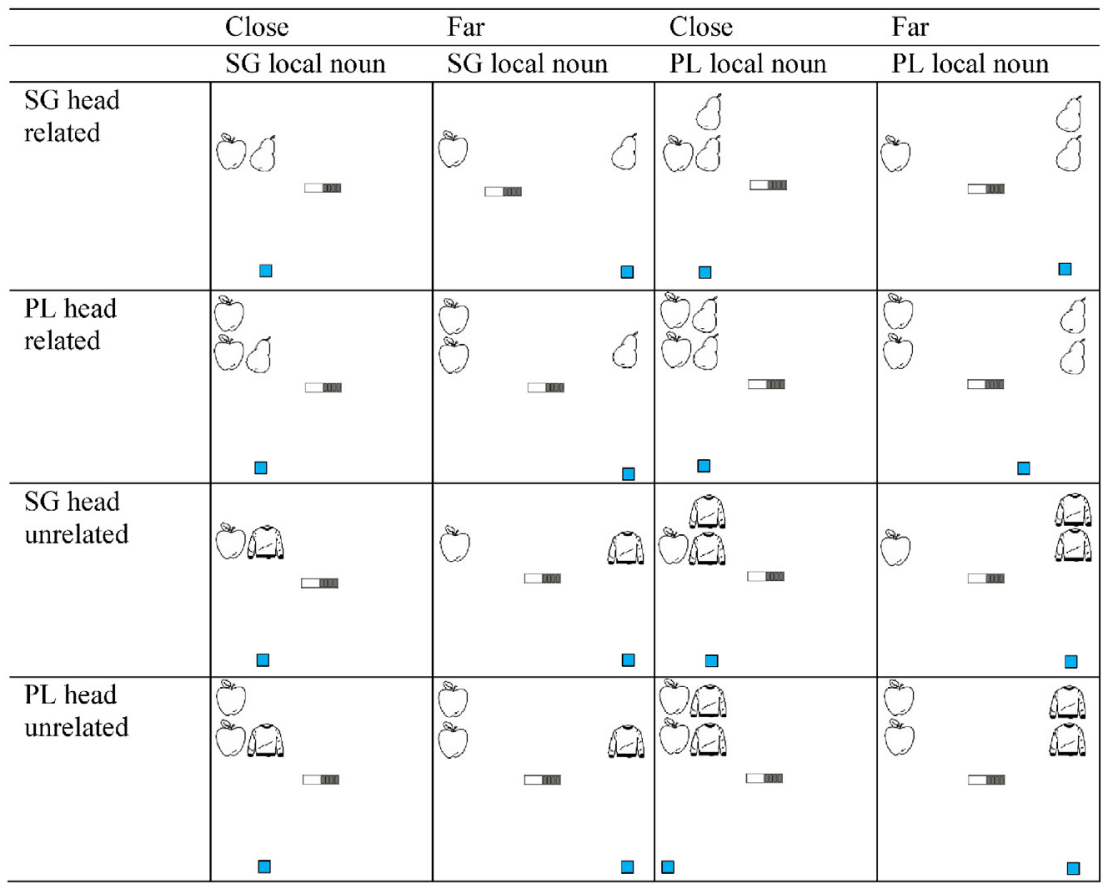

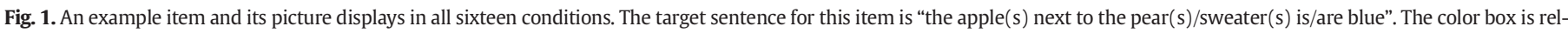

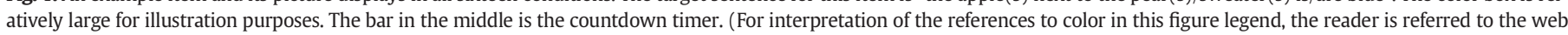
version of this article.)

\subsection{Procedure}

Participants were tested individually in a quiet room. At the beginning of the session, they were given a booklet with the pictures used in the experiment with their names written underneath. To reduce the rate of naming errors, participants were asked to study the names for the pictures and use them in the experiment.

The experiment started with a practice block of 20 trials, followed by four experimental blocks of 56 trials each. Participants were allowed to take short breaks between blocks. The experiment lasted approximately 30 minutes.

On each practice and experimental trial, a fixation cross appeared in the top left corner of the screen for 500 ms or until the drift correction was performed. This fixation cross served as a fixation point for the drift correction and to make sure that the participants' first gaze would be on the location of the head noun picture. After a blank screen of $150 \mathrm{~ms}$, the pictures appeared, with the head noun picture always appearing in the top left corner in the location of the fixation cross. Along with the pictures, a small timer appeared in the center of the screen. The timer counted down in eight steps from 2.5 seconds to zero. Participants were instructed to try to complete their description before the timer ran out. Trials proceeded automatically with intertrial intervals of $500 \mathrm{~ms}$.

\subsection{Scoring and analyses}

Participants' responses were recorded, transcribed and scored for non-agreement errors (e.g., wrong words, numbers, or incomplete responses) and agreement errors. Agreement errors were included in the analysis only if the remainder of the sentence was correct.

Speech onset latencies were measured with Praat speech analysis software (Boersma \& Weenink, 2010). Each sentence started with the same determiner (de), allowing this determiner to be planned and produced before the rest of the noun phrase was encoded. Onsets for the determiner might therefore not be informative about the planning strategy employed. As such, speech onsets were coded at the first noun rather than the determiner. In addition, onset times for the local noun and the verb were determined.

Finally, eye movements were used to identify the gaze patterns and determine the first pass gaze duration for the head noun picture. In order to quantify participants' eye movements and fixation patterns, interest areas were drawn around the pictures, approximately one $\mathrm{cm}$ larger than the size of the pictures. First pass gaze durations were calculated by subtracting the start time of the eye gaze from the end time of the eye gaze to an interest area, such that both fixations and saccades within one interest area would be included.

Statistical analyses were run using linear mixed effects regression models with crossed effects of subjects and items using the lme4 package in R (Bates, 2005; R Development Core Team, 2011). Factors were centered before entering the models treating participants and items as random effects (Baayen, 2008). Backwards elimination was used for the fixed effects, starting with a full model and leaving out nonsignificant interactions. Models were run with the maximal random structure justified by the data when possible (Barr, Levy, Scheepers, \& Tily, 2013). List number was included initially as a factor in the statistical models, but as it did not contribute significantly to the models, it was left out of the models presented here.

The first set of analyses assessed the amount of parallel activation in each condition. The first analysis investigated the effects of the spatial configuration and the semantic similarity of the pictures on speech onset latencies for the first noun. Due to rightward skewing, speech onsets were natural log-transformed before analysis. Variables included were Head Noun Number (coded as -1 for singular, and 1 for plural), Mismatch (coded as -1 for matching number, and 1 for mismatching number), Spatial Distance (coded as -1 for the Far condition, and 1 for the Close condition), Semantic Similarity (coded as -1 for unrelated pictures, and 1 for related pictures), and Block (coded as -2 through 2 for blocks 1 to 4 ). The inclusion of random slopes in the analysis meant that resampling methods for calculating statistical probability were not available. In accordance with Baayen (2008), absolute t-values exceeding 2 were interpreted as statistically significant. The second analysis 
investigated the effects of spatial configuration of the pictures and the semantic similarity between them on gaze durations for the first picture. This analysis paralleled the analysis of the speech onset times.

A second set of analyses concerned the error rates in the experiment. Here, mixed-effects models used a logistic linking function (Jaeger, 2008). The first analysis compared the rates of non-agreement errors across experimental conditions. The second analysis investigated whether attraction occurred in the agreement error rates. Variables included in both analyses were Head Noun Number, Mismatch, and Block.

The final and critical analysis investigated the effects of the spatial configuration of the pictures and the semantic similarity between them on agreement error rates. Because of the extremely low error rates in the matching condition (see Results), only mismatching trials were included in this analysis. Trials in which the head noun picture was fixated between production of the local noun and the verb were excluded. The variables included were Head Noun Number, Block, Spatial Distance, Semantic Similarity, and the interaction between Spatial Distance and Semantic Similarity. Because of its importance with regard to our experimental hypotheses, this interaction remained in all analyses regardless of whether it was significant or not.

\section{Results}

\subsection{Speech onset latencies}

Speech onsets for invalid and incorrect trials and those more than three standard deviations above the participants' mean were excluded from the analysis $(n=933,17.4 \%)$. Planning two nouns in parallel was predicted to lead to longer speech onsets than when those two nouns were planned more sequentially. Additionally, previous research suggests that semantic similarity should lead to longer speech onsets for related than for unrelated nouns.

Fig. 2 shows the speech onset times, with results confirming the predictions above. Statistical analysis revealed main effects of Spatial Distance and Semantic Similarity (see Table 1). Participants were slower to initiate their responses when the pictures were close relative to when they were far apart, suggesting that the Close condition encouraged parallel processing of the pictures. Additionally, participants were slower when the pictures were related compared to when they were unrelated, indicating semantic interference. There was an interaction between Semantic Similarity and Spatial Distance. Follow-up analyses showed that in the Close condition, speech onsets were slower for related rela-

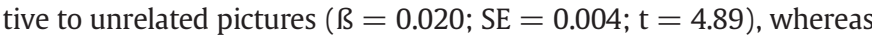
no semantic interference was observed in the Far condition $(ß=-0.002 ; \mathrm{SE}=0.005 ; \mathrm{t}=-0.36)$.

There was also a three-way interaction between Mismatch, Semantic Similarity, and Block, see Fig. 3. This interaction came from the fact that the magnitude of the semantic interference effect decreased over

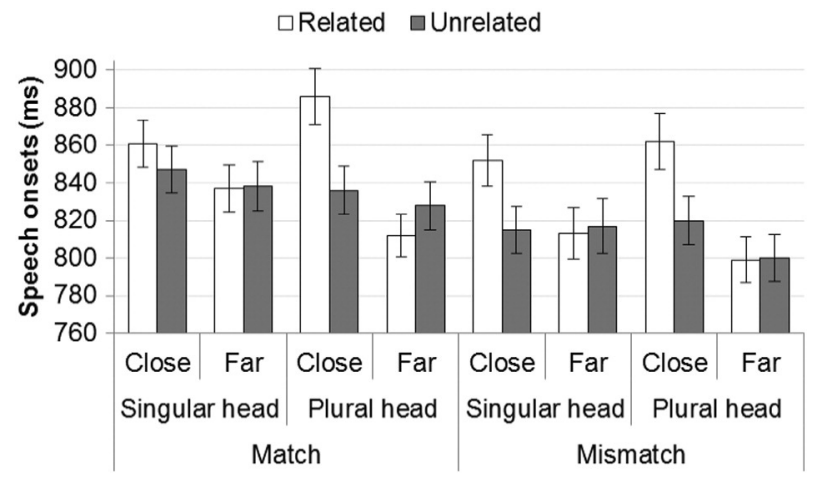

Fig. 2. Average speech onset times for items with singular and plural heads in close and far conditions with related and unrelated word pairs in matching and mismatching conditions. Error bars represent SE of the mean across participants for illustrative purposes.
Table 1

Mixed-effects model predicting speech onset times.

\begin{tabular}{lclll}
\hline Variable & Coefficient & SE & \multicolumn{1}{l}{ t } & Random Slope \\
\hline (Intercept) & 6.705 & 0.032 & 209.13 & Subjects, items \\
Head noun number & -0.002 & 0.003 & -0.63 & Subjects, items \\
Mismatch & -0.005 & 0.004 & -1.39 & Subjects, items \\
Spatial distance & 0.017 & 0.004 & 3.90 & Subjects, items \\
Semantic similarity & 0.010 & 0.004 & 2.70 & Subjects, items \\
Block & -0.005 & 0.004 & -1.26 & Subjects, items \\
Similarity * distance & 0.011 & 0.003 & 4.00 & Subjects \\
Mismatch $*$ similarity * block & -0.006 & 0.002 & -3.30 & \\
\hline
\end{tabular}

Note. Coefficients correspond to natural log-transformed speech onsets.

the course of the experiment in the mismatching condition $(\Omega=-0.006 ; \mathrm{SE}=0.003 ; \mathrm{t}=-2.44)$, but increased in the matching condition $(~ B=0.005 ; \mathrm{SE}=0.002 ; \mathrm{t}=2.25$ ). Specifically, in the matching condition, the onsets for related condition remained stable ( $\beta=0.001 ; \mathrm{SE}=0.005 ; \mathrm{t}=0.18$ ), whereas the onsets for the unrelated

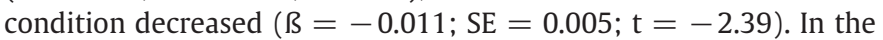
mismatching condition the speech onset latencies for the related condition decreased $(\beta=0.013 ; \mathrm{SE}=0.006 ; \mathrm{t}=-2.21)$, whereas the onsets for the unrelated condition remained fairly stable $(\beta=0.001$; SE $=$ 0.006 ; $t=0.19$, see Fig. 3 ). One has to keep in mind, though, that these interactions are collapsed across the close and far conditions, making the interference effects rather small to begin with. No effects of Head Noun Number or Mismatch were found.

In sum, the speech onsets suggested that parallel activation occurred mostly in the Close condition. Converging evidence for this interpretation is provided by the eye-tracking measures below.

\subsection{Eye movements}

Prior to analyzing people's gaze durations, we first examined whether participants re-fixated the first picture due to interference from the mismatching local noun. As many earlier studies have shown that speakers typically look at pictures in the order they will mention them (e.g., Griffin, 2001; Griffin \& Bock, 2000; Meyer, Sleiderink, \& Levelt, 1998), the predicted pattern was that participants would first look at the head noun picture, followed by the local noun picture, and finally at the color box. In the majority of trials, participants indeed started with a head-local-color pattern (89.4\%). On the remaining trials, sometimes no fixations were recorded to the head noun picture $(0.3 \%)$, to the local noun picture $(7.1 \%)$, or to the color box $(0.7 \%)$, most often because the participants fixated just outside of the interest area. On many trials the participants looked back to the head or local noun picture after their first inspection of the three areas of interest (77.3\%). These second gazes typically occurred well after the onset of the spoken utterances.

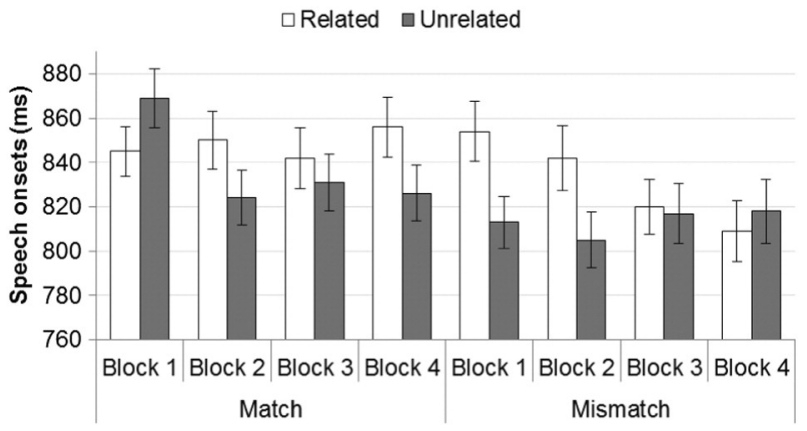

Fig. 3. Average speech onset times across different blocks with related and unrelated word pairs in matching and mismatching conditions. Error bars represent SE of the mean across participants for illustrative purposes. 


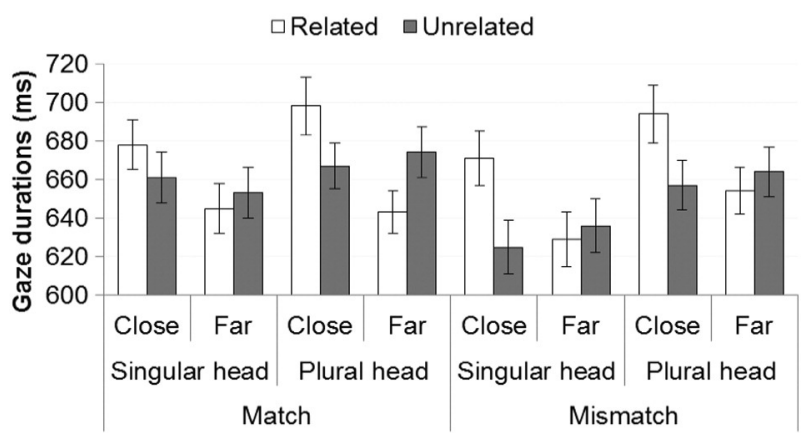

Fig. 4. Average gaze durations for items with singular and plural heads in close and far conditions with related and unrelated word pairs in matching and mismatching conditions. Error bars represent SE of the mean across participants for illustrative purposes.

Second, gaze durations for the first looks at the head noun picture in all conditions were measured. Longer gaze durations have been shown to indicate semantic interference. The gaze durations are shown in Fig. 4. Trials that were discarded from the speech onset latency analyses were also discarded from this analysis.

Statistical analysis revealed a main effect of Head Noun Number (see Table 2). Participants looked longer at the first picture when a plural than when a singular form was required. Similar to the speech onsets, there was an interaction between Semantic Similarity and Spatial Distance in the gaze durations. Follow-up analyses showed that in the Close condition, participants looked longer at the first picture when it was semantically related to the second picture than when it was unrelated ( $\beta=0.017 ; \mathrm{SE}=0.008 ; \mathrm{t}=2.11$ ), whereas in the Far condition, gazes were longer in the unrelated condition than in the related condition ( $\beta=-0.014 ; \mathrm{SE}=0.007 ; \mathrm{t}=-2.14$ ). Examination of Fig. 4 shows that this facilitation effect was only an effect of differential processing of the semantically-related items between the close and far condition $(B=0.026$; $S E=0.008 ; t=3.20)$, and no effect of spatial distance was observed for semantically-unrelated items $(~ B=-0.006$; $\mathrm{SE}=$ $0.007 ; \mathrm{t}=-1.00$ ). Also similar to the speech onsets, there was a three-way interaction between Mismatch, Semantic Similarity and Block, which came from the fact that semantic interference slightly decreased over the course of the experiment in the mismatching condition $(B=-0.006 ; \mathrm{SE}=0.004 ; \mathrm{t}=-1.38)$ and increased in the matching condition $(~ B=0.009 ; \mathrm{SE}=0.004 ; \mathrm{t}=1.99)$. A similar interaction was found between Head Noun Number, Semantic Similarity and Block. Semantic interference decreased across blocks in plural head condition ( $\beta=-0.005 ; \mathrm{SE}=0.004 ; \mathrm{t}=-1.14)$, but increased in singular head condition ( $\beta=0.007$; $S E=0.005 ; t=1.64$ ). It should be noted that the effect of Block was not significant in either of the interactions; thus the interactions between Head Noun Number, Semantic Similarity and Block, and between Mismatch, Semantic Similarity and Block were driven by the crossing over of the directions of the Block effects.

Results from both speech onsets and gaze durations demonstrated evidence of semantic interference in the Close condition. In both cases,

Table 2

Mixed-effects model predicting gaze durations.

\begin{tabular}{lclrl}
\hline Variable & Coefficient & SE & \multicolumn{1}{l}{ t } & Random slope \\
\hline (Intercept) & 6.445 & 0.029 & 220.58 & Subjects, items \\
Head noun number & 0.014 & 0.007 & 2.03 & Subjects, items \\
Mismatch & -0.001 & 0.006 & -0.21 & Subjects, items \\
Spatial distance & 0.010 & 0.007 & 1.47 & Subjects, items \\
Semantic similarity & 0.002 & 0.007 & 0.35 & Subjects \\
Block & 0.006 & 0.005 & 1.10 & Subjects \\
Similarity $*$ distance & 0.016 & 0.005 & 3.39 & Subjects \\
Mismatch $*$ similarity * block & -0.006 & 0.003 & -2.07 & \\
Head $*$ similarity * block & -0.008 & 0.003 & -2.66 & \\
\hline
\end{tabular}

Note. Coefficients correspond to natural log-transformed gaze durations. the semantic interference effect decreased over the course of the experiment, possibly due to the repetition of the materials. These results confirm that parallel activation was more likely in the Close condition than in the Far condition.

\subsection{Error rates}

Non-agreement errors ( $\mathrm{n}=512,8.5 \%$ ) included incorrect head nouns, prepositions, local nouns, or colors, incorrect numbers for the head noun or local noun, or incomplete utterances. The error rates per condition are shown in Fig. 5.

More non-agreement errors were made when the head noun was plural than when it was singular, and when the nouns mismatched than when they matched, as indicated by main effects of Head Noun Number and Mismatch (see Table 3). There was an interaction between Mismatch and Spatial Distance, which meant that within the Mismatch condition, marginally more errors were made for spatially close pictures than for far pictures $(~(=-0.14 ; \mathrm{SE}=0.07 ; \mathrm{z}=-1.93 ; \mathrm{p}=.053$ ), whereas there was no effect of distance in the Matching condition $(~(B=0.12 ; \mathrm{SE}=0.09 ; \mathrm{z}=1.37 ; \mathrm{p}=.172$ ). There was no effect of semantic relatedness. Interpreting this pattern of results is difficult because the absolute number of non-agreement errors was small and because the category comprised different types of errors with different origins. However, comparing the results for the non-agreement errors and for the speech onset latencies, we can note that there is no evidence for a speed-accuracy trade-off.

As agreement errors can only be established in sentences that are otherwise correct, non-agreement errors were removed from subsequent analyses. After removal of these errors each cell in the design still included at least 319 observations. The following analysis was meant to confirm that attraction occurred, with the prediction that there would be more agreement errors in the mismatching condition than in the matching condition.

As Fig. 6 shows, the error rates in the matching condition were extremely low. The statistical analysis revealed main effects of Mismatch and Block (see Table 4). More agreement errors were made when the number of the local noun mismatched with the number of the head noun compared to when they matched, confirming that attraction occurred. The main effect of Block came from error rates decreasing over the course of the experiment. Replicating previous studies (Bock \& Miller, 1991; Eberhard, 1997; Veenstra, Acheson, \& Meyer, 2014), there was also an interaction between Head Noun Number and Mismatch, which came from the fact that the attraction was stronger for the singular heads $(~ B=2.17$; $S=0.35 ; z=6.81 ; p<0.001$ ) than for the plural heads $(B=1.22$; $\mathrm{SE}=0.20 ; \mathrm{z}=5.97 ; \mathrm{p}<0.001$ ), although it was significant in both conditions.

Our primary research question concerned the magnitude of the attraction effect, which is only found in the mismatching condition.

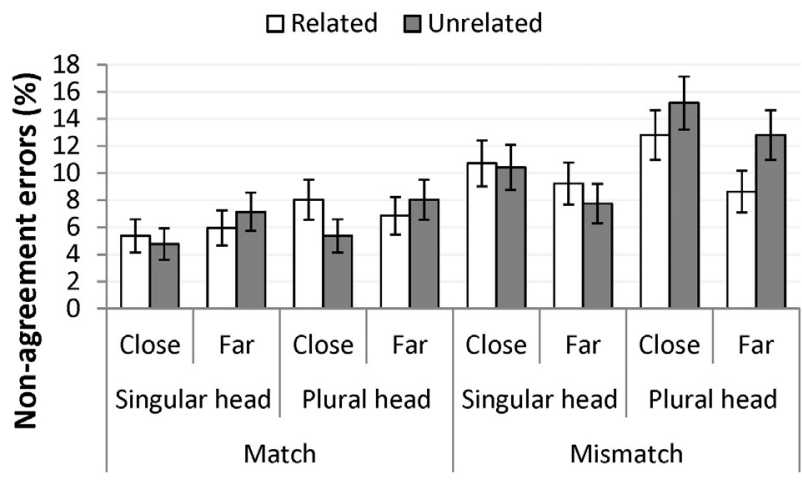

Fig. 5. Non-agreement error rates for items with singular and plural heads in close and far conditions with related and unrelated word pairs in matching and mismatching conditions. Error bars represent the SE of the mean across participants for illustrative purposes. 
Table 3

Logistic Mixed-Effects Model predicting Non-Agreement Errors.

\begin{tabular}{lccrll}
\hline Variable & Coefficient & SE & z-Value & $\operatorname{Pr}(>|z|)$ & Random slope \\
\hline (Intercept) & -2.724 & 0.158 & -17.21 & $<.001$ & Subjects, items \\
Head noun number & 0.220 & 0.068 & 3.23 & $<.01$ & Subjects, items \\
Mismatch & 0.361 & 0.066 & 5.47 & $<.001$ & Subjects, items \\
Spatial distance & -0.019 & 0.062 & -0.31 & .758 & Subjects, items \\
Semantic similarity & -0.059 & 0.064 & -0.92 & .360 & Subjects, items \\
Block & -0.049 & 0.042 & -1.17 & .243 & Subjects, items \\
Similarity $*$ distance & -0.029 & 0.050 & -0.58 & .565 & \\
Mismatch $*$ distance & -0.123 & 0.052 & -2.36 & $<.05$ & \\
\hline
\end{tabular}

Note. Coefficients correspond to Logits.

Given that there were very few errors in the matching condition (ranging from a single error to a maximum of seven errors in a cell of the experimental design), the following analysis was restricted to the mismatching condition only. The lexical interference account predicts stronger attraction in the Close condition, where the nouns are more likely to be planned in parallel than in the Far condition.

Trials in which participants looked back at the head noun picture after producing the local noun, but before producing the verb, were removed from the analysis $(n=110,4.6 \%)$. On these trials, participants might have been able to check for the number of the head noun, thereby reducing the chance of an agreement error (note, however, that analyses including these trials showed similar patterns to the analyses presented here). Fig. 7 shows the agreement error rates in the mismatching conditions.

The statistical analysis revealed only a main effect of Block (see Table 5). Participants made fewer attraction errors over the course of the experiment (18\% in block $1,13 \%$ in block $2,10 \%$ in block 3 , and $12 \%$ in block 4). The main effects of Head Noun Number and Spatial Distance approached significance. There was a trend of more errors being made for singular head noun items than for plural head noun items. In addition, marginally more errors were made when the pictures were far apart than when they were close together. There was no effect of Semantic Similarity.

The current results suggest that agreement errors were not affected by the spatial distance and semantic similarity manipulations. If anything, the effects were opposite from the direction predicted by a lexical interference account as more errors should have been observed in the Close condition, rather than in the Far condition.

\section{Discussion}

According to the lexical interference account of attraction proposed by Solomon and Pearlmutter (2004); see also Gillespie \& Pearlmutter,

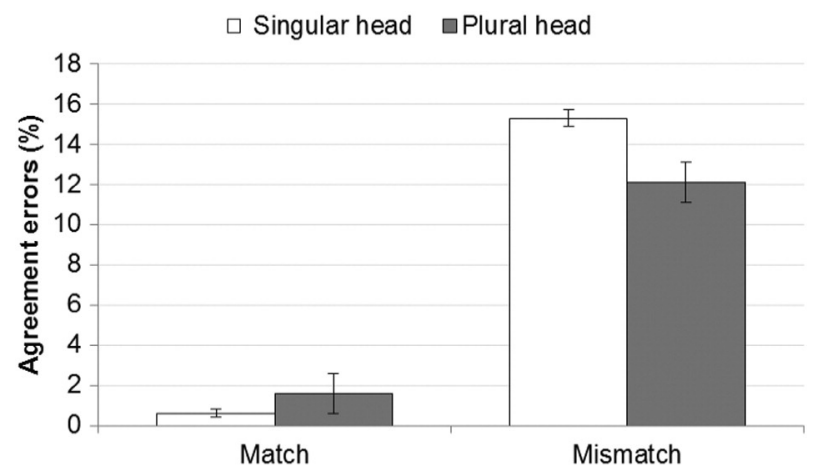

Fig. 6. Agreement error rates for items with singular and plural heads in matching and mismatching conditions. Error bars represent the SE of the mean across participants for illustrative purposes.
Table 4

Logistic Mixed-Effects Model predicting Attraction.

\begin{tabular}{lccrrl}
\hline Variable & Coefficient & SE & z-Value & $\operatorname{Pr}(>|z|)$ & Random slope \\
\hline (Intercept) & -3.75 & 0.22 & -17.10 & $<.001$ & Subjects, items \\
Head noun number & 0.19 & 0.14 & 1.31 & .192 & Subjects \\
Mismatch & 1.59 & 0.19 & 8.42 & $<.001$ & Subjects \\
Block & -0.15 & 0.05 & -3.12 & .002 & Subjects \\
Head Number * mismatch & -0.34 & 0.12 & -2.80 & .005 & \\
\hline
\end{tabular}

Note. Coefficients correspond to Logits.

2011b) parallel planning of head and local noun in the subject noun phrase leads to simultaneous activation of their number features. Such simultaneous activation in turn leads to more number interference and, consequently, more agreement errors relative to when nouns are planned sequentially (Gillespie \& Pearlmutter, 2011b; Solomon \& Pearlmutter, 2004). In order to encourage speakers to process the objects mentioned in the subject noun phrase in parallel or more sequentially, we varied the spatial distance between them.

To assess whether our spatial distance manipulation influenced the degree of parallel processing of the objects and their names, we measured speech onsets and gaze durations. We had two ways to establish parallel planning. First, we hypothesized that presenting the pictures in a spatially close configuration would encourage parallel activation as the local noun picture should be extrafoveally processed while the head noun picture was fixated. In a far configuration, parallel activation would be less likely, as the pictures were too far apart to be processed simultaneously. Therefore, we predicted longer speech onsets and gaze durations in the close condition than in the far condition. A main effect of spatial distance was indeed seen in the speech onsets, but was not reliable in the gaze durations.

The second way of establishing parallel activation was through observing effects of semantic interference. Semantic similarity between two objects to be named in succession can only affect the processing time for the object to be named first if there is some temporal overlap in the processing of the two objects. Therefore, we expected to see evidence of semantic interference when objects were close together (and likely to be processed in parallel) but not when they were far apart. In both speech onsets and gaze durations, there was a significant interaction between semantic similarity and spatial distance. As predicted, in the close condition speech onset latencies and gaze durations were longer for related than for unrelated objects, whereas no such effect was observed in the Far condition. Taken together, these findings suggest that parallel activation was indeed more likely in the Close than in the Far condition. This confirms earlier findings of extrafoveal processing of objects in object naming studies (e.g., Malpass \& Meyer, 2010; Meyer et al., 2008). Our finding that extrafoveal processing

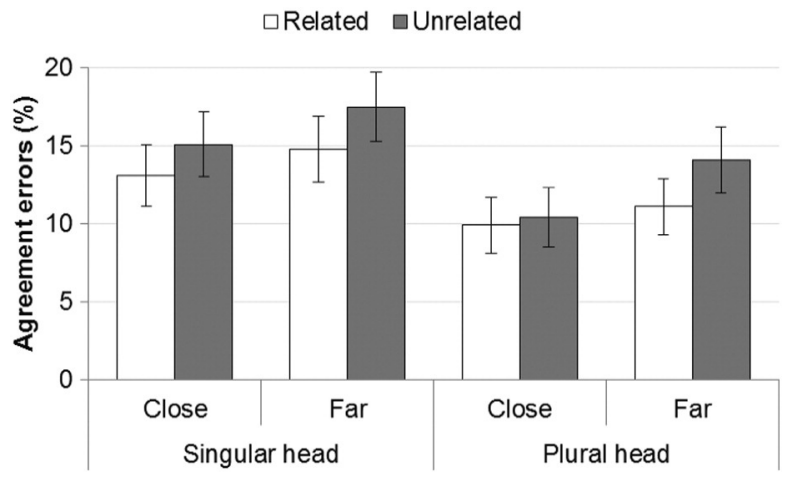

Fig. 7. Agreement errors rates for items with singular and plural heads in close and far conditions with related and unrelated word pairs (mismatching conditions only). Error bars represent SE of the mean across participants for illustrative purposes. 
Table 5

Logistic mixed-effects model predicting agreement errors.

\begin{tabular}{lllrrl}
\hline Variable & Coefficient & SE & z-Value & $\operatorname{Pr}(>|z|)$ & Random slope \\
\hline (Intercept) & -2.281 & 0.211 & -10.801 & $<.001$ & Subjects, items \\
Head noun number & -0.198 & 0.110 & -1.794 & .073 & Subjects, items \\
Spatial distance & -0.152 & 0.083 & -1.842 & .066 & Subjects, items \\
Semantic similarity & -0.112 & 0.075 & -1.493 & .135 & Subjects, items \\
Block & -0.168 & 0.053 & -3.184 & .001 & Subjects, items \\
Similarity * distance & 0.020 & 0.067 & 0.296 & .767 & \\
\hline
\end{tabular}

Note. Coefficients correspond to Logits.

depends on spatial distance is consistent with the preliminary results reported by Meyer and Konopka (2011), who argued that the spatial configuration of objects influenced the likelihood of parallel processing and planning of their names.

It is important to note that although the predicted patterns of the semantic relatedness manipulation-semantic interference-was observed in speech onset times, gaze durations showed an unexpected pattern. As predicted, semantic interference was observed for semantically related items in the Close condition. In contrast, in the Far condition, we observed semantic facilitation for semantically related items. This pattern of results suggests that, contrary to our expectations, parallel processing was not completely eliminated in the far condition, and some shallow or slow processing of the distant second picture occurred while participants were looking at the first picture. However, this processing must have been qualitatively different from the way near pictures were processed as the semantic effect on the gaze durations reversed from interference to facilitation. Such a finding is not unprecedented in the field, and is in line with work by Roelofs and Piai (Roelofs, 2003; Roelofs \& Piai, 2013, 2015), who argue that semantic relatedness can lead to facilitation when the lemma for a competitor is activated but has not yet been selected (see also Chen \& Mirman, 2012 for a similar account). Presumably facilitation can occur if some of the semantic features of semantically related objects are processed in parallel. In the current study extrafoveal processing of the second picture could have activated semantic features for the head noun, facilitating lexical access.

In this context, it is important to highlight that in the materials used in the present study (as in the study by Meyer \& Konopka, 2011) the semantically related items tended to be visually similar as well (see Table A2). This was confirmed in a rating study with 83 participants, who were asked rate each combination of pictures for semantic and visual similarity on a 5-point-scale. Both similarity scores were significantly higher for the related than for the unrelated items (semantic similarity: related $(\mathrm{m}=4.88, \mathrm{SD}=0.15)$ vs. unrelated $(\mathrm{m}=1.36, \mathrm{SD}=0.37), \mathrm{t}(55)=63.94, \mathrm{p}<.001$; visual similarity: related $(\mathrm{m}=3.18, \mathrm{SD}=0.98)$ vs. unrelated $(\mathrm{m}=1.75, \mathrm{SD}=0.42), \mathrm{t}(55)=$ $10.44, \mathrm{p}<.001$ ), and the indicators for the two types of similarity were highly correlated $(r=.70)$. This raises the question whether our interference effect stemmed from parallel visual processing. Additional analyses on a subset of items that had a visual similarity rating below 3 in the related condition (47\% of the data) yielded significant effects in the RTs similar to the analyses reported above, but the effects of semantic similarity in the gaze durations were, although in the same directions as before, no longer significant. Nevertheless, if the interference effect in the Close condition was entirely visual, it should also have been eliminated from the RTs. Furthermore, regression analyses treating the visual similarity ratings as a factor showed that for RTs, there were no significant effects of Visual Similarity, while keeping the effects of Semantic Similarity and Spatial Distance intact. For the gaze durations however, the visual similarity ratings interacted with semantic similarity $(\beta=0.028, S E=0.010, t=2.82)$, meaning that for related items visual similarity increased the gaze duration $(\beta=0.057, \mathrm{SE}=0.013$, $\mathrm{t}=4.54)$, whereas for the unrelated items visual similarity did not have a significant effect $(\beta=-0.030, \mathrm{SE}=0.020, \mathrm{t}=-1.53)$. These effects were independent of the spatial distance, and show that the semantic interference effects were not purely visual.

There is some evidence from other studies using pictures that semantic interference has a lexical basis, whereas semantic facilitation has an early visual-conceptual basis. For instance, Aristei et al. (2012) simultaneously presented two pictures, which both had to be named in a novel compound. They found a clear semantic interference effect for related pictures, although they did not control for visual similarity. A study that did control for visual similarity was carried out by Jescheniak, Matushanskaya, Mädebach, and Müller (2014). They asked participants to name the blue picture in an array of two simultaneously presented pictures. The pictures were preceded by a visual cue (an arrow pointing to either the target or the distractor). When the distractor was cued, they found semantic interference between related pictures (see Humphreys, Lloyd-Jones, Fias, \& 1995, for similar results using a post-cuing paradigm). In these studies, the distractor picture had to be processed up to the lexical level. Similarly, La Heij, Heikoop, Akerboom, and Bloem (2003) found semantic interference in speech onset latencies when two related pictures were presented together for at least $300 \mathrm{~ms}$ (replicating Glaser \& Glaser, 1989). However, in a second experiment, where the distracter was only presented for $50 \mathrm{~ms}$, the semantic interference effect reversed into facilitation. Due to this brief presentation, the distracter pictures might not have been processed up to the lexical level, thereby yielding facilitation rather than interference. This ties in with other studies finding facilitation (preview benefits) from visually related distractor pictures (e.g., Pollatsek, Rayner, \& Collins, 1984; Schotter, Ferreira, \& Rayner, 2013). In fact, it has been argued that the small effects of facilitation stemming from visualconceptual overlap can be overruled by the stronger interference effects stemming from lexical-semantic overlap (e.g., Abdel Rahman \& Melinger, 2009; Aristei et al., 2012, Navarrete \& Costa, 2005). Following these results, one could argue that the interference effect we found in the Close condition results from parallel processing of both visual and lexical information (with strong interference overruling weak facilitation), whereas the small facilitatory effect in the Far condition results from parallel processing of visual information alone.

Our data suggests that some amount of parallel planning occurred at both spatial distances. Importantly, however, the degree of parallel planning was different between the close and far conditions. In the condition of primary interest, namely the head and local noun mismatch condition, we saw substantial semantic interference for the speech onset latencies and gaze durations in the close conditions (39 ms and $42 \mathrm{~ms}$, respectively), and weak semantic facilitation effects in the far condition ( 3 and $8 \mathrm{~ms}$, respectively, which were not significant). This pattern of results implies that we are still able to address the primary research question of the current study, namely, whether the degree of parallel planning of the objects mentioned in the subject noun phrase is related to the likelihood of agreement errors.

The lexical interference account predicts stronger attraction for mismatching nouns planned in parallel than for nouns planned sequentially. Thus, if parallel planning increases attraction, higher error rates should be seen in the Close condition relative to the Far condition. However, our results did not confirm this prediction. There was a marginal effect of Spatial Distance in the opposite direction: More errors were made in the Far condition relative to the Close condition.

It is important to note that we did find a mismatch effect on error rates, even though it was not modulated by spatial distance. This typical error pattern shows that the current paradigm did tap into agreement processes. As often reported in the agreement literature, the attraction effect was asymmetrical with regard to the number of the head noun. More errors were found for singular heads combined with plural local nouns compared to plural heads combined with singular local nouns. This asymmetry has been explained by the markedness of plural forms, which allows marked plural local nouns to exert attraction on singular head nouns, but rarely the reverse (Bock \& Eberhard, 1993; 
Eberhard, 1997). Nevertheless, there is precedence to find significant attraction after plural head nouns, particularly in paradigms using picture naming instead of sentence completion (e.g., Veenstra, Acheson, \& Meyer, 2014).

Though the main aim of the study was to assess the relationship between the time course of the processing of the subject noun phrase and the likelihood of agreement errors, it is worth highlighting that Mismatch affected the rates of agreement errors, as expected, but not the speech onset latencies or gaze duration for the first object. One might have expected otherwise, especially for the Close condition. Since there was a semantic interference effect, one might expect to see a grammatical number mismatch effect as well, with slower processing for objects and nouns mismatching in number compared to those matching in number. However, no such number mismatch effect was found. This result is consistent with data from the picture-word interference paradigm. Schiller and Caramazza (2002) found semantic interference for semantically related pictures and words, but no effect of mismatching number. Similarly, our results showed similar speech onsets for the number matching condition $(\mathrm{m}=843 \mathrm{~ms} ; \mathrm{SD}=225 \mathrm{~ms})$, and the mismatching condition ( $\mathrm{m}=822 \mathrm{~ms}$; $\mathrm{SD}=208$ ), as well as similar gaze durations for the matching condition $(\mathrm{m}=672 \mathrm{~ms}$; $\mathrm{SD}=242 \mathrm{~ms}$ ), and the mismatching condition ( $\mathrm{m}=662 \mathrm{~ms}$; $\mathrm{SD}=221 \mathrm{~ms}$ ). This suggests that a mismatch in number and the associated morphological markers of head and local nouns does not delay the selection or morpho-phonological encoding of the nouns (or anything related to the inflection of verbs, for that matter).
In contrast to the lack of a number interference effect, semantic similarity did lead to interference in the current study. The classic account of semantic interference is based on mutual activation between related concepts and lemmas (e.g., Levelt et al., 1999; Roelofs, 1992). Co-activation arises because there are "links" in the conceptual and lemma stratum. Whereas the semantic links are present between related concepts and lemmas a priori, number features may only be activated ad hoc based on the conceptual information of the specific utterance. Thus, number links are not present in the lexicon. It is therefore not surprising to find semantic interference but not number interference in our latency measures.

To conclude, the hypothesis, derived from Gillespie and Pearlmutter's (2011b) and Solomon and Pearlmutter's (2004) earlier work, that more parallel processing of the components of the subject noun phrase would be associated with a stronger attraction effect was not supported. Although we have evidence that we induced more parallel planning in the Close than in the Far condition, we did not find evidence for increased lexical number interference in the former condition. Instead, our results suggest that temporal overlap in the generation of the components of a complex noun phrase does not have a large effect on the ease of generating subject-verb agreement. This could mean that the number feature for the inflected verb was determined as soon as first picture was identified, or that it was only set after the planning of the subject noun phrase had been completed. Further research is needed to corroborate the current findings and to work towards a detailed processing model for the generation of subject-noun agreement.

\section{Appendix A}

Table A1

Log-transformed form frequencies of the head and local nouns.

\begin{tabular}{|c|c|c|c|c|c|}
\hline Head noun & Singular & Plural & Local noun & Singular & Plural \\
\hline Koe (cow) & 6.422 & 6.729 & Geit (goat) & 5.094 & 5.476 \\
\hline Auto (car) & 8.854 & 7.410 & Bus (bus) & 7.295 & 5.403 \\
\hline Appel (apple) & 5.717 & 5.533 & Peer (pear) & 5.247 & 5.142 \\
\hline Broek (pants) & 7.768 & 5.485 & Trui (sweater) & 6.402 & 4.522 \\
\hline Tafel (table) & 8.991 & 6.457 & Stoel (chair) & 8.509 & 7.080 \\
\hline Kikker (frog) & 5.215 & 5.147 & Slak (snail) & 4.205 & 5.063 \\
\hline Wortel (carrot) & 6.295 & 6.690 & Tomaat (tomato) & 4.595 & 5.663 \\
\hline Kaars (candle) & 5.999 & 6.080 & Lamp (lamp) & 6.772 & 6.073 \\
\hline Leeuw (lion) & 6.463 & 5.753 & Tijger (tiger) & 5.403 & 4.248 \\
\hline Lepel (spoon) & 6.174 & 4.615 & Vork (fork) & 6.052 & 4.263 \\
\hline Trommel (drums) & 6.410 & 5.176 & Gitaar (guitar) & 5.384 & 3.761 \\
\hline Vlieg (fly) & 5.956 & 5.900 & Bij (bee) & - & 5.727 \\
\hline Boot (boat) & 7.643 & 5.740 & Kano (canoe) & 4.796 & 4.344 \\
\hline Sjaal (scarf) & 5.598 & 4.060 & Muts (hat) & 5.501 & 3.989 \\
\hline
\end{tabular}

Note. The CELEX database did not contain the frequency for the singular form of bij.

Table A2

Related noun pairs (and their ratings).

\begin{tabular}{|c|c|c|c|c|c|c|c|c|c|c|}
\hline & \multicolumn{5}{|c|}{ Visual similarity } & \multicolumn{5}{|c|}{ Semantic similarity } \\
\hline & SG-SG & SG-PL & PL-PL & PL-SG & & SG-SG & SG-PL & PL-PL & PL-SG & \\
\hline Koe/geit & 4.25 & 3.58 & 4.57 & 3.82 & 4.06 & 4.94 & 4.88 & 5 & 4.81 & 4.91 \\
\hline Auto/bus & 2.73 & 2.8 & 3.53 & 3 & 3.02 & 4.93 & 4.82 & 4.94 & 4.72 & 4.85 \\
\hline Appel/peer & 3.26 & 4.2 & 4.2 & 4.15 & 3.95 & 4.88 & 4.94 & 5 & 4.94 & 4.94 \\
\hline Broek/trui & 1.81 & 2.07 & 2.95 & 2.07 & 2.23 & 4.94 & 5 & 4.81 & 5 & 4.94 \\
\hline Tafel/stoel & 2.24 & 2.65 & 2.76 & 2.5 & 2.54 & 4.74 & 4.94 & 4.82 & 4.75 & 4.81 \\
\hline Kikker/slak & 2.4 & 2.55 & 2.88 & 2.5 & 2.58 & 4.6 & 4.72 & 4.38 & 4.47 & 4.54 \\
\hline Wortel/tomaat & 1.26 & 2.29 & 2.27 & 2.4 & 2.06 & 4.47 & 4.88 & 4.8 & 4.81 & 4.74 \\
\hline Kaars/lamp & 2.65 & 3.1 & 3.63 & 3.67 & 3.26 & 4.81 & 4.69 & 5 & 4.94 & 4.86 \\
\hline Leeuw/tijger & 4.4 & 3.71 & 4.38 & 3.89 & 4.10 & 5 & 4.88 & 5 & 4.88 & 4.94 \\
\hline Lepel/vork & 4.07 & 4.19 & 4 & 4.1 & 4.09 & 5 & 5 & 4.94 & 4.94 & 4.97 \\
\hline Trommel/gitaar & 1.38 & 1.6 & 2.4 & 1.47 & 1.71 & 4.65 & 4.93 & 4.94 & 5 & 4.88 \\
\hline Vlieg/bij & 4.47 & 4.71 & 4.93 & 4.6 & 4.68 & 4.94 & 4.94 & 5 & 5 & 4.97 \\
\hline Boot/kano & 3.95 & 3.75 & 4.1 & 3.6 & 3.85 & 4.94 & 5 & 4.88 & 5 & 4.96 \\
\hline \multirow[t]{2}{*}{ Sjaal/muts } & 1.59 & 2.6 & 2.63 & 2.6 & 2.36 & 5 & 4.88 & 5 & 4.94 & 4.96 \\
\hline & 2.89 & 3.13 & 3.52 & 3.17 & 3.18 & 4.85 & 4.89 & 4.89 & 4.87 & 4.88 \\
\hline
\end{tabular}


Table A3

Unrelated noun pairs (and their ratings).

\begin{tabular}{|c|c|c|c|c|c|c|c|c|c|c|}
\hline & \multicolumn{5}{|c|}{ Visual similarity } & \multicolumn{5}{|c|}{ Semantic similarity } \\
\hline & SG-SG & SG-PL & PL-PL & PL-SG & & SG-SG & SG-PL & PL-PL & PL-SG & \\
\hline Koe/bus & 1.59 & 1.87 & 2.1 & 1.87 & 1.86 & 1.41 & 1.27 & 1.56 & 1.33 & 1.39 \\
\hline Auto/geit & 1.41 & 2.05 & 2.27 & 1.52 & 1.81 & 1.19 & 1.19 & 1.33 & 1.25 & 1.24 \\
\hline Appel/trui & 1.4 & 2.5 & 2.58 & 2.52 & 2.25 & 1 & 1.58 & 1.56 & 1.35 & 1.37 \\
\hline Broek/peer & 1.33 & 1.56 & 2.5 & 1.88 & 1.82 & 1.06 & 1.5 & 1.35 & 1.25 & 1.29 \\
\hline Tafel/slak & 1.14 & 1.53 & 1.85 & 1.53 & 1.51 & 1.05 & 1.27 & 1.19 & 1.27 & 1.20 \\
\hline Kikker/stoel & 1.37 & 2 & 2 & 1.8 & 1.79 & 1 & 1.13 & 1.07 & 1 & 1.05 \\
\hline Wortel/lamp & 1.47 & 2.09 & 2 & 2.15 & 1.93 & 1.07 & 1.06 & 1.38 & 1 & 1.13 \\
\hline Kaars/tomaat & 1.07 & 1.59 & 2.18 & 1.36 & 1.55 & 1.33 & 1 & 1.56 & 1.33 & 1.31 \\
\hline Leeuw/vork & 1.14 & 1.53 & 1.7 & 1.53 & 1.48 & 1.06 & 1.07 & 1.19 & 1.07 & 1.10 \\
\hline Lepel/tijger & 1.12 & 1.71 & 1.75 & 1.65 & 1.56 & 1.06 & 1.24 & 1.07 & 1 & 1.09 \\
\hline Trommel/bij & 1.1 & 1.79 & 2.14 & 1.71 & 1.69 & 1.24 & 1.81 & 1.41 & 2.06 & 1.63 \\
\hline Vlieg/gitaar & 1.13 & 2.09 & 2.42 & 1.73 & 1.84 & 1.07 & 1.35 & 1.94 & 1.29 & 1.41 \\
\hline Boot/muts & 1.3 & 1.33 & 1.82 & 1.16 & 1.40 & 1.88 & 2.88 & 1.47 & 2.38 & 2.15 \\
\hline \multirow[t]{2}{*}{ Sjaal/kano } & 1.33 & 2.5 & 2.06 & 2.24 & 2.03 & 1.4 & 1.63 & 2.25 & 1.47 & 1.69 \\
\hline & 1.28 & 1.87 & 2.10 & 1.76 & 1.75 & 1.20 & 1.43 & 1.45 & 1.36 & 1.36 \\
\hline
\end{tabular}

\section{References}

Abdel Rahman, R., \& Melinger, A. (2009). Semantic context effects in language production: A swinging lexical network proposal and a review. Language and Cognitive Processes, 24, 713-734

Aristei, S., Zwitserlood, P., \& Abdel Rahman, R. (2012). Picture-induced semantic interference reflects lexical competition during object naming. Frontiers in Psychology, 3, 28.

Baayen, R. H. (2008). Analyzing linguistic data: A practical introduction to statistics. Cambridge: Cambridge University Press.

Baayen, R. H., Piepenbrock, R., \& van Rijn, H. (1993). The \{CELEX\} lexical data base on $\{C D-R O M\}$.

Barr, D. J., Levy, R., Scheepers, C., \& Tily, H. J. (2013). Random effects structure for confirmatory hypothesis testing: Keep it maximal. Journal of Memory and Language, 68(3), 255-278.

Bates, D. M. (2005). Fitting linear mixed models in R: Using the lme4 package. R News: The Newsletter of the R Project, 5(1), 27-30

Belke, E., Meyer, A. S., \& Damian, M. F. (2005). Refractory effects in picture naming as assessed in a semantic blocking paradigm. The Quarterly Journal of Experimental Psychology, 58(4), 667-692.

Bock, K., \& Eberhard, K. M. (1993). Meaning, sound and syntax in English number agreement. Language and Cognitive Processes, 8(1), 57-99.

Bock, K., \& Miller, C. A. (1991). Broken agreement. Cognitive Psychology, 23(1), 45-93.

Bock, K., Eberhard, K. M., Cutting, J. C., Meyer, A. S., \& Schriefers, H. (2001). Some attractions of verb agreement. Cognitive Psychology, 43(2), 83-128.

Boersma, P., \& Weenink, D. (2010). Praat: Doing phonetics by computer [computer program], Version 5.1. 44

Brehm, L., \& Bock, K. (2013). What counts in grammatical number agreement? Cognition, 128(2), 149-169.

Chen, Q., \& Mirman, D. (2012). Competition and cooperation among similar representations: Toward a unified account of facilitative and inhibitory effects of lexical neighbors. Psychological Review, 119(2), 417.

Dell, G. S. (1986). A spreading activation theory of retrieval in sentence production. Psychological Review, 93, 283-321.

Eberhard, K. M. (1997). The marked effect of number on subject-verb agreement. Journal of Memory and Language, 36(2), 147-164.

Francis, W. S., Corral, N. I., Jones, M. L., \& Sáenz, S. P. (2008). Decomposition of repetition priming components in picture naming. Journal of Experimental Psychology: General, 137(3), 566-590.

Franck, J., Vigliocco, G., \& Nicol, J. (2002). Subject-verb agreement errors in French and English: The role of syntactic hierarchy. Language and Cognitive Processes, 17(4), 371-404.

Freedman, M. L., Martin, R. C., \& Biegler, K. (2004). Semantic relatedness effects in conjoined noun phrase production: Implications for the role of short-term memory. Cognitive Neuropsychology, 21(2-4), 245-265.

Gillespie, M., \& Pearlmutter, N. J. (2011a). Effects of semantic integration and advance planning on grammatical encoding in sentence production. In L. Carlson, C. Hoelscher, \& T. F. Shipley (Eds.), Proceedings of the 33rd annual conference of the cognitive science society (pp. 1625-1630). Austin, TX: Cognitive Science Society.

Gillespie, M., \& Pearlmutter, N. J. (2011b). Hierarchy and scope of planning in subject-verb agreement production. Cognition, 118(3), 377-397.

Glaser, W. R., \& Glaser, M. O. (1989). Context effects in stroop-like word and picture processing. Journal of Experimental Psychology: General, 118(1), 13

Griffin, Z. M. (2001). Gaze durations during speech reflect word selection and phonological encoding. Cognition, 82(1), B1-B14.

Griffin, Z. M., \& Bock, K. (2000). What the eyes say about speaking. Psychological Science, $11(4), 274-279$.

Haskell, T. R., Thornton, R., \& MacDonald, M. C. (2010). Experience and grammatical agreement: Statistical learning shapes number agreement production. Cognition, 114(2), 151-164
Humphreys, K. R., Boyd, C. H., \& Watter, S. (2010). Phonological facilitation from pictures in a word association task: Evidence for routine cascaded processing in spoken word production. The Quarterly Journal of Experimental Psychology, 63(12), 2289-2296.

Humphreys, G. W., Lloyd-Jones, T. J., \& Fias, W. (1995). Semantic interference effects on naming using a postcue procedure: Tapping the links between semantics and phonology with pictures and words. Journal of Experimental Psychology: Learning Memory, and Cognition, 21(4), 961.

Jaeger, T. F. (2008). Categorical data analysis: Away from ANOVAs (transformation or not) and towards logit mixed models. Journal of Memory and Language, 59(4), 434-446.

Jescheniak, J. D., Matushanskaya, A., Mädebach, A., \& Müller, M. M. (2014). Semantic interference from distractor pictures in single-picture naming: Evidence for competitive lexical selection. Psychonomic Bulletin \& Review, 21(5), 1294-1300.

Konopka, A. E. (2012). Planning ahead: How recent experience with structures and words changes the scope of linguistic planning. Journal of Memory and Language, 66(1), 143-162.

La Heij, W., Heikoop, K. W., Akerboom, S., \& Bloem, I. (2003). Picture naming in picture context: Semantic interference or semantic facilitation? Psychology Science, 45(1), 49-62.

Levelt, W. J. M., Roelofs, A., \& Meyer, A. S. (1999). A theory of lexical access in speech production. Behavioral and Brain Sciences, 22, 1-75.

Mädebach, A., Jescheniak, J. D., Oppermann, F., \& Schriefers, H. (2011). Ease of processing constrains the activation flow in the conceptual-lexical system during speech planning. Journal of Experimental Psychology: Learning, Memory, and Cognition, 37(3), 649-660.

Malpass, D., \& Meyer, A. S. (2010). The time course of name retrieval during multipleobject naming: Evidence from extrafoveal-on-foveal effects. Journal of Experimental Psychology: Learning, Memory, and Cognition, 36(2), 523.

Martin, R. C., Crowther, J. E., Knight, M., Tamborello, F. P., \& Yang, C. L. (2010). Planning in sentence production: Evidence for the phrase as a default planning scope. Cognition 116(2), 177-192.

Meyer, A. S., \& Konopka, A. E. (2011). Predictors of sequential object naming: visual layout and working memory capacity. Paper presented at the 52nd meeting of the psychonomic society. Seattle: US.

Meyer, A. S., Ouellet, M., \& Häcker, C. (2008). Parallel processing of objects in a naming task. Journal of Experimental Psychology: Learning, Memory, and Cognition, 34(4), 982.

Meyer, A. S., Sleiderink, A. M., \& Levelt, W. J. (1998). Viewing and naming objects: Eye movements during noun phrase production. Cognition, 66(2), B25-B33.

Meyer, A. S., van der Meulen, F., \& Brooks, A. (2004). Eye movements during speech planning: Talking about present and remembered objects. Visual Cognition, 11(5), 553-576.

Morgan, J. L., van Elswijk, G., \& Meyer, A. S. (2008). Extrafoveal processing of objects in a naming task: Evidence from word probe experiments. Psychonomic Bulletin \& Review, 15(3), 561-565.

Navarrete, E., \& Costa, A. (2005). Phonological activation of ignored pictures: Further evidence for a cascade model of lexical access. Journal of Memory and Language, 53(3), 359-377.

Oppermann, F., Jescheniak, J. D., \& Schriefers, H. (2010). Phonological advance planning in sentence production. Journal of Memory and Language, 63(4), 526-540.

R Development Core Team (2011). R: A Language and Environment for Statistical Computing. Vienna: R Foundation for Statistical Computing.

Pollatsek, A., Rayner, K., \& Collins, W. E. (1984). Integrating pictorial information across eye movements. Journal of Experimental Psychology: General, 113(3), 426.

Roelofs, A. (1992). A spreading-activation theory of lemma retrieval in speaking. Cognition, 42(1), 107-142.

Roelofs, A. (2003). Goal-referenced selection of verbal action: Modeling attentional control in the stroop task. Psychological Review, 110, 88-125.

Roelofs, A., \& Piai, V. (2013). Associative facilitation in the stroop task: Comment on Mahon et al. (2012). Cortex, 49, 1767-1769.

Roelofs, A., \& Piai, V. (2015). Aspects of competition in word production: Reply to Mahon and Navarrete (2014). Cortex, 64, 420-424.

Schiller, N. O., \& Caramazza, A. (2002). The selection of grammatical features in word production: The case of plural nouns in German. Brain and Language, 81(1-3), 342-357. 
Schotter, E. R., Ferreira, V. S., \& Rayner, K. (2013). Parallel object activation and attentional gating of information: Evidence from eye movements in the multiple object naming paradigm. Journal of Experimental Psychology: Learning, Memory, and Cognition, 39(2), 365-374.

Severens, E., Lommel, S. V., Ratinckx, E., \& Hartsuiker, R. J. (2005). Timed picture naming norms for 590 pictures in Dutch. Acta Psychologica, 119(2), 159-187.

Smith, M., \& Wheeldon, L. (2004). Horizontal information flow in spoken sentence production. Journal of Experimental Psychology: Learning Memory, and Cognition, 30(3), 675.

Solomon, E. S., \& Pearlmutter, N. J. (2004). Semantic integration and syntactic planning in language production. Cognitive Psychology, 49(1), 1-46.

Veenstra, A., \& Acheson, D. J. (2015). Semantic integration and subject-verb agreement: Independent effects of notional and grammatical number. (Manuscript submitted for publication).
Veenstra, A., Acheson, D. J., \& Meyer, A. S. (2014a). Keeping it simple: Studying grammatical encoding with lexically reduced item sets. Frontiers in Psychology, 5, 783.

Veenstra, A., Acheson, D. J., Bock, K., \& Meyer, A. S. (2014b). Effects of semantic integration on the production of subject-verb agreement: Evidence from Dutch. Language, Cognition and Neuroscience, 29(3), 355-380.

Vigliocco, G., Butterworth, B., \& Semenza, C. (1995). Constructing subject-verb agreement in speech: The role of semantic and morphological factors. Journal of Memory and Language, 34(2), 186-215. 